\title{
CONCIRCULAR TRANSFORMATIONS OF RIEMANNIAN MANIFOLDS
}

\author{
JACQUELINE FERRAND
}

Introduction. In this paper we deal with connected Riemannian manifolds $(M, g),(\bar{M}, \bar{g})$ of dimension $n$ and of class $C^{3}$ at least. We shall denote their sectional curvature tensors by $R, \bar{R}$, their Ricci tensors by $r, \bar{r}$, and their scalar curvatures by $S, \bar{S}$. The space of $C^{2}$-vector fields on $M$ will be denoted by $\Gamma(M)$.

With every strictly positive scalar function $u$ of class $C^{2}$ on $M$, we associate the conformal deformation $g \mapsto u^{-2} g$ of $(M, g)$. The curvature tensors of $\left(M, \bar{g}=u^{-2} g\right)$ are then given, in local coordinates, by:

$$
\begin{gathered}
\bar{r}_{i j}-r_{i j}=\frac{n-2}{u} u_{i, j}+\left(\frac{\Delta u}{u}-(n-1) \frac{\operatorname{grad}^{2} u}{u^{2}}\right) g_{i j}, \\
u^{2} \bar{R}_{i j k l}-R_{i j k l}=-\frac{\operatorname{grad}^{2} u}{u^{2}}\left(g_{i k} g_{j i}-g_{i l} g_{j k}\right) \\
+\frac{1}{u}\left(g_{i k} u_{j, l}+g_{j l} u_{i, k}-g_{i l} u_{j, k}-g_{j k} u_{i, l}\right), \\
u^{-2} \bar{S}-S=2(n-1)\left(\frac{\Delta u}{u}-\frac{n}{2} \frac{\operatorname{grad}^{2} u}{u^{2}}\right),
\end{gathered}
$$

where the $u_{i, j}$ are the covariant derivatives of second order of $u$, and $\Delta u=g^{i j} u_{i, j}$. (These formulae follow from the usual ones by setting $u=e^{-\sigma}$.)

Definition. The scalar function $u$ and the associated conformal deformation $g_{\mapsto u^{-2}} g$ are said to be concircular if there exists a scalar function $\varrho$ such that

$$
u_{i, j}=\varrho g_{i j}(i, j=1,2 \ldots n) .
$$

Then, by setting $\tau=\varrho / u-\left(1 /\left(2 u^{2}\right)\right) \operatorname{grad}^{2} u$, the formulae $(0.1),(0.2),(0.3)$ reduce to:

$$
\begin{gathered}
\bar{r}_{i j}-r_{i j}=2(n-1) \tau g_{i j}, \\
u^{2} \bar{R}_{i j k l}-R_{i j k l}=2 \tau\left(g_{i k} g_{j l}-g_{i l} g_{j k}\right), \\
u^{-2} \bar{S}-S=2 n(n-\cdot 1) \tau .
\end{gathered}
$$

More generally, if $(M, g)$ and $(\bar{M}, \bar{g})$ are two Riemannian manifolds of the same dimension $n$, a morphism $f: M \rightarrow \bar{M}$ is said to be concircular if it is conformal and if there exists a scalar function $\varrho$ on $M$ such that $u=\left|f^{\prime}\right|^{-1}$ satisfy (0.4). In other 
words $f:(M, g) \rightarrow(\bar{M}, \bar{g})$ is concircular if, and only if, $g_{\mapsto} f^{*} \bar{g}$ is a concircular deformation of $(M, g)$.

A conformal morphism $f$ such that $\left|f^{\prime}\right|$ is constant will be called a similarity; a conformal deformation $g \mapsto u^{-2} g$ such that $u$ is constant will be said to be a homothety.

Concircular transformations have been introduced by K. Yano [13] as conformal transformations preserving geodesic circles (curves whose normal parametrizations satisfy $d^{3} x / d s^{3}=k(d x / d s)$ with $k=$ const.). Later, W. O. Vogel [12] proved that every morphism preserving geodesic circles is necessarily conformal; and $\mathrm{Y}$. Tashiro [9] gave a classification of complete Riemannian manifolds admitting a concircular field $u$ (i.e. satisfying (0.4) but not necessarily $>0$ ).

Let us notice that the characterization of the sphere given by M. Obata [8] sets upon this classification. For other papers relative to concircular transformations, see $[5,8,10]$. An equation close to $(0.4)$ has been studied by $\mathbf{J}$. Lafontaine [7].

In this paper, we first review the main results concerning concircular deformations (Section 1); then we shall set some apparently new results, and examine the special case $\tau=0$ (Sections 2, 3, 4). Most part of these results are extensible to pseudo-Riemannian manifolds.

1. Preliminaries. At firs $t$ let us notice that, if $n \geqq 3$, either relation $(0.5),(0.6)$ involves $(0.4)$ with $\varrho=\tau u+(1 /(2 u)) \operatorname{grad}^{2} u$. It easily follows :

Property 1.1. Any conformal mapping between Einstein manifolds of dimension $n \geqq 3$ is concircular.

Conversely if $f:(M, g) \rightarrow(\bar{M}, \bar{g})$ is concircular, and if $(M, g)$ is an Einstein space [resp. a space with constant sectional curvature], then so is $(\bar{M}, \bar{g})$.

Property 1.2. If $u: M \rightarrow \boldsymbol{R}$ is concircular (satisfying (0.4)) then:

a) For any constant $\lambda, u+\lambda$ is concircular; and $u^{-1}$ is concircular on $\left(M, u^{-2} g\right)$.

b) There exists locally a function $G$ such that

$$
\operatorname{grad}^{2} u=G(u) \text { and } \varrho=\frac{1}{2} G^{\prime}(u) .
$$

c) For any vector fields $X, Y$ on $M$

$$
R(X, Y) \cdot \operatorname{grad} u=\left(L_{X} \varrho\right) Y-\left(L_{Y} \varrho\right) X .
$$

Proof. The first assertion a) is obvious; for $n \geqq 3$ the second one can be obtained by exchanging $g$ and $\bar{g}$.

To prove b), we notice that, with intrinsic notations, (0.4) is equivalent with

$$
\forall X \in \Gamma(M): \quad \nabla_{X} \operatorname{grad} u=\varrho X
$$

which implies

$$
\nabla_{X} \operatorname{grad}^{2} u=2 \varrho X \cdot \operatorname{grad} u=2 \varrho \nabla_{X} u
$$

or, in other terms, $d\left(\operatorname{grad}^{2} u\right)=2 \varrho d u$. 
Finally (1.2) follows from (1.3) by covariant differentiation.

Property 1.3. In order that $u: M \rightarrow \boldsymbol{R}$ be concircular, it is necessary and sufficient that there exist local coordinates $\left(x_{i}\right)$, with $x_{1}=u$, such that the metric of $M$ be

$$
d s^{2}=\frac{d u^{2}}{G(u)}+G(u) \sum_{i, j \geqq 2} \gamma_{i j}\left(x_{2}, \ldots, x_{n}\right) d x_{i} d x_{j}
$$

with $\varrho=(1 / 2) G^{\prime}(u)$, or equivalently:

$$
d s^{2}=d v^{2}+\varphi^{2}(v) \sum_{i, j \geqq 2} \gamma_{i j}\left(x_{2}, \ldots, x_{n}\right) d x_{i} d x_{j}
$$

with $v=\int[G(u)]^{-1 / 2} d u$ and $\varphi^{2}(v)=G(u)$.

Obviously, the function $G(u)=\operatorname{grad}^{2} u$ need not be defined at stationary points of $u$; but on a complete manifold, there are at most two such points (cf. [10]).

For the applications of concircular properties to Einstein or other special spaces, see [2], [5], [9], [10], [11], [12], [13].

2. Conformal properties. From the results reviewed in Section 1, we easily infer

Lemma 2.1. If $u: M \rightarrow \boldsymbol{R}$ is concircular (i.e. satisfies (0.4)), then

a) the $u$-hypersurfaces (defined by $u=$ Const.) are totally umbilical, of constant normal curvature $\varrho /|\operatorname{grad} u|$.

b) the integral curves of $\operatorname{grad} u$ are geodesics whose tangent at any point is an eigen direction of the Ricci tensor.

The proof is classical; the last assertion follows from (1.2). In [4] we proved that the "conformal circles" of E. Cartan and K. Yano are the curves which, by a suitable conformal deformation of $M$ can be changed into geodesics whose tangent is an eigen direction of the Ricci tensor. By looking for a converse of Lemma 2.1, we obtain:

Theorem 2.2. Let $(M, g)$ be a Riemannian manifold and $u: M \rightarrow \boldsymbol{R}$ a strictly positive scalar function having only isolated stationary points. In order that there exists a metric $\bar{g}$, conformal to $g$, such that $u$ be concircular on $(M, \bar{g})$, it is necessary and sufficient that

i) the u-hypersurfaces be totally umbilical,

ii) their orthogonal trajectories be conformal circles.

Proof. The necessity of these conditions follows from Lemma 2.1, since they are invariant under a conformal deformation.

Conversely we know (cf. [3]) that the condition i) implies the existence of local coordinates $x_{i}$, with $x_{1}=u$, such that the metric of $M$ is

$$
d s^{2}=A^{2} d u^{2}+B^{2} \sum_{i, j \geqq 2} \gamma_{i j}\left(x_{2}, \ldots, x_{n}\right) d x_{i} d x_{j} .
$$

If the condition ii) is satisfied, the curves $x_{i}=$ Const. $(i \geqq 2)$ are still conformal 
circles for the metric $d s^{2} / A^{2}$; and since they are geodesics for this new metric, $\partial / \partial u$ must be an eigen vector for the Ricci tensor. Now the $(1, j)$-components $(j \neq 1)$ of the Ricci tensor for $d s^{2} / A^{2}$ are given by

$$
R_{1 j}=-(n-2) \frac{\partial^{2}}{\partial u \partial x_{j}} \log \left(\frac{B}{A}\right)
$$

and therefore the condition ii) is equivalent with the existence of two positive functions $\alpha, \beta$ such that

$$
\frac{B}{A}=\alpha(u) \beta\left(x_{2}, \ldots, x_{n}\right) .
$$

By setting $\bar{\gamma}_{i j}=\beta \gamma_{i j}$, we have

$$
\frac{d s^{2}}{A^{2}}=d u^{2}+\alpha^{2}(u) \sum_{i, j \geq 2} \bar{\gamma}_{i j}\left(x_{2}, \ldots, x_{n}\right) d x_{i} d x_{j}
$$

and the Property (1.3) shows that $u$ is concircular for the metric $d s^{2} /\left(\alpha A^{2}\right)$.

Let us notice also that $\int \alpha d u$ is concircular for $d s^{2} / A^{2}$. On the other hand we have:

Lemma 2.3. If $u$ is concircular on $(M, g)$, and if $\theta$ is a differentiable function on $u(M)$, the only metrics conformally equivalent with $g$ which admit $\theta(u)$ as a concircular field are given by $\bar{g}=C\left|\theta^{\prime}\right| g$, with $C=$ Const.

Application: Conformally flat manifolds admitting a concircular deformation. Starting with $\boldsymbol{R}^{n}$ we have to look for functions $u>0$ such that the $u$-hypersurfaces be totally umbilical and their orthogonal trajectories be conformal circles: in other words, $u$-hypersurfaces are hyperspheres (or hyperplanes) whose orthogonal trajectories are circles (or straight lines); and they must belong to a bundle of spheres or hyperplanes. By using a Möbius transformation, we are brought back to the three typical bundles, respectively defined by

$$
\text { i) } \Sigma x_{i}^{2}=\text { Const., ii) } x_{1}=\text { Const., iii) } \frac{x_{2}}{x_{1}}=\text { Const. }
$$

Therefore, if $u$ is concircular on a conformally flat manifold $(M, g)$ there exist local coordinates $x_{i}$ and a function $\theta$ of one variable such that

$$
u=\theta\left(\Sigma x_{i}^{2}\right), \quad u=\theta\left(x_{1}\right) \quad \text { or } \quad u=\theta\left(x_{2} / x_{1}\right)
$$

and, for a suitable choice of the function $\sigma\left(x_{1}, \ldots, x_{n}\right)$ :

$$
d s^{2}=e^{2 \sigma} \sum d x_{i}^{2} .
$$

Now it can be directly checked that $\Sigma d x_{i}^{2}$ admits $x_{1}$ and $\Sigma x_{i}^{2}$ for concircular 
fields, while $\left(x_{1}^{2}+x_{2}^{2}\right)^{-1} \Sigma d x_{i}^{2}$ admits $\operatorname{arctg}\left(x_{2} / x_{1}\right)$ as a concircular field. With help of Lemma 2.3, we can state:

Theorem 2.4. The conformally flat metrics admitting a concircular field are locally given, by suitable choice of coordinates, by

or

$$
d s^{2}=\varphi\left(\Sigma x_{i}^{2}\right) \Sigma d x_{i}^{2}, \quad d s^{2}=\varphi\left(x_{1}\right) \Sigma d x_{i}^{2}
$$

$$
d s^{2}=\left(x_{1}^{2}+x_{2}^{2}\right)^{-1} \varphi\left(\operatorname{arctg} x_{2} / x_{1}\right) \Sigma d x_{i}^{2}
$$

where $\varphi$ is an arbitrary function of one variable; and the associated concircular fields are the primitives of $\varphi$.

This result completes Theorem 2 of [6]. Let us notice that, by setting $u=$ $\operatorname{arctg}\left(x_{2} / x_{1}\right)$ and $v=(1 / 2) \log \left(x_{1}^{2}+x_{2}^{2}\right)$, the metrics of the third type can also be written

$$
d s^{2}=\varphi(u)\left(d u^{2}+d v^{2}+e^{-2 v} \sum_{i \geqq 3} d x_{i}^{2}\right) .
$$

The global existence of concircular fields depends on the topology of the manifold.

3. A special case: quasi-similarities. Definition 3.1. A conformal deformation [resp. a conformal morphism f] will be called quasi-homothetic [resp. a quasisimilarity] if there exists a scalar function @ such that the associated function $u$ [resp. the function $\left.u=\left|f^{\prime}\right|^{-1}\right]$ satisfies

$$
u_{i, j}=\varrho g_{i j} \text { and } \operatorname{grad}^{2} u=2 \varrho u \text {. }
$$

(In other terms: $u$ is concircular and the associated function $\tau=\varrho / u-\left(1 /\left(2 u^{2}\right)\right) \operatorname{grad}^{2} u$ is null.)

If $n \geqq 3$, the conditions 3.1 express that the Ricci and sectional curvatures of $M$ are transformed in the same way as under an homothety [resp. a similarity] of ratio $u^{-1}$ (see formulae $(0.5)$ and $(0.6)$ ). In particular, any conformal morphism of Ricci-flat manifolds (i.e. with Ricci curvature zero) of dimension $n \geqq 3$ is automatically a quasi-similarity. More precisely, we have:

Lemma 3.1. Let $(M, g)$ be a flat [resp. Ricci-flat] manifold of dimension $n \geqq 3$, and $f:(M, g) \rightarrow(\bar{M}, \bar{g})$ a conformal morphism. In order that $(f(M), \bar{g})$ be flat [resp. Ricci-flat] it is necessary and sufficient that $f$ be a quasi-similarity.

Quasi-similarities have been studied by us in [5]. Let us recall some results.

Lemma 3.2. If $u=M \rightarrow \boldsymbol{R}$ satisfies (3.1), then $\varrho$ is a constant; and for any vector fields $X, Y$ on $M$, we have

$$
R(X, Y) \operatorname{grad} u=0,
$$


Lemma 3.3. In order that $u: M \rightarrow \boldsymbol{R}$ be quasi-homothetic, it is necessary and sufficient that there exists a constant $\varrho$ and local coordinates $\left(x_{i}\right)$, with $x_{1}=u$, such that:

$$
d s^{2}=\frac{d u^{2}}{2 \varrho u}+u \sum_{i, j \geqq 2} \gamma_{i j}\left(x_{2}, \ldots, x_{n}\right) d x_{i} d x_{j} .
$$

(This is a special case of Property 1.3.)

For brevity, we shall say that a quasi-homothety [resp. a quasi-similarity] is proper if the associated function $u$ is not constant.

Theorem 3.4. A complete Riemannian manifold does not admit any proper quasi-homothety or quasi-similarity.

Proof. On any geodesic $\gamma$ satisfying $d x / d s=\operatorname{grad} u /|\operatorname{grad} u|$, we have $d u / d s=$ $(2 \varrho u)^{1 / 2}$ and $d^{2} u / d s^{2}=\varrho$; hence, if $\varrho \neq 0: 2 u=\varrho\left(s-s_{0}\right)^{2}$; and if $s$ could run from $-\infty$ to $+\infty, u$ would take the value zero.

The case of $\boldsymbol{R}^{n}$ and of conformally flat manifolds. First of all, on $\boldsymbol{R}^{n}$, the only nonconstant solutions of (3.1) are the functions

$$
u=\frac{1}{2} \varrho \Sigma\left(x_{i}-a_{i}\right)^{2} \quad\left(\varrho, a_{i}=\text { Const. }\right)
$$

This easy remark provides a very short proof of the theorem of Liouville in class $C^{3}$ : namely, if $U$ is an open set of $\boldsymbol{R}^{n}(n \geqq 3)$ and if $f: U \rightarrow \boldsymbol{R}^{n}$ is a conformal (not necessarily injective) morphism, then, from Lemma 3.1, $f$ is a quasi-similarity and $u=\left|f^{\prime}\right|^{-1}$ satisfies (3.4) for some values of $\varrho, a_{1}, \ldots, a_{n}$. If $\varrho=0, u=$ Const. and $f$ is a similarity. If $\varrho \neq 0$, let be $j$ the inversion $x \mapsto a+(2 / \varrho)|x-a|^{-2}(x-a)$. Then we have $\left|f^{\prime}(x)\right|=\left|j^{\prime}(x)\right|=\left|j^{\prime}(j(x))\right|^{-1}$, hence $\left|(f \circ j)^{\prime}(x)\right|=1$ and $f \circ j$ is an isometry. In both cases $f$ is a Möbius transformation.

On another side, we may complete Lemma 2.3 and Theorem 2.4 by stating

Lemma 3.5. If $u$ is quasi-homothetic for $(M, g)$, the only metrics, conformally equivalent with $g$, which admit a nonconstant function $\theta(u)$ as quasi-homothetic, are of the type $\bar{g}=C u^{\lambda-1} g$ with $C, \lambda=$ Const., $\lambda \neq 0$, the function $\theta$ then being $\theta(u)=k u^{\lambda}$ ( $k=$ Const.).

Theorem 3.6. The conformally flat metrics admitting a quasi-homothetic deformation are locally given, with suitable coordinates, by:

$$
\begin{gathered}
d s^{2}=\left[\Sigma\left(x_{i}^{2}\right)\right]^{\lambda-1} \Sigma d x_{i}^{2}, \quad d s^{2}=e^{\lambda x_{1}} \Sigma d x_{i}^{2} \quad \text { or } \\
d s^{2}=e^{\lambda u}\left(d u^{2}+d v^{2}+e^{-2 v} \sum_{i \geqq 3} d x_{i}^{2}\right)
\end{gathered}
$$

where $\lambda \neq 0$ is a constant.

Among these metrics there is no one of constant curvature $\neq 0$, as we could infer from (3.2). 
4. Concircular deformations of submanifolds. The restriction to a submanifold of a concircular transformation is still conformal, but not necessarily concircular. In fact we have:

Theorem 4.1. Let $u: M \rightarrow \boldsymbol{R}$ concircular. In order that the restriction of $u$ to a submanifold $V$ be concircular it is necessary and sufficient that at any point of $V$ either grad $u$ be tangent to $V$

or the normal component $N$ of $\operatorname{grad} u$ be an umbilical direction for $V$.

Proof. We know that $v=u_{\mid V}$ is concircular if, and only if, there exists a function $\sigma$ on $V$ such that

$$
\forall X \in \Gamma(V): \quad \bar{\nabla}_{X}(\operatorname{grad} v)=\sigma X,
$$

where $\bar{\nabla}_{X}$ is the induced connection on $V$. Now $\operatorname{grad} v=T=\operatorname{grad} u-N$ is the tangential component of $\operatorname{grad} u$, and $\bar{\nabla}_{X} T$ is the tangential component of $\nabla_{X} T$. By using (1.3) we see that the condition (4.1) is realized if, and only if, $\nabla_{X} N-(\varrho-\sigma) X$ is normal to $V$; and, if $N \neq 0$, this means that $N$ is an umbilical direction for $V$.

This last condition is realized, in particular, if grad $u$ is normal to $V$ at any point; in that case $v$ is constant and $\sigma=0$.

If $u$ is quasi-homothetic (which implies $\operatorname{grad}^{2} u=2 \varrho u$ and $\varrho=$ Const.) and if we want $v=u_{\mid V}$ to be quasi-homothetic, we have to set the additional condition $\operatorname{grad}^{2} v=$ $2 \sigma v$ with $\sigma=$ Const. This is realized if and only if $|T|^{2} /|N|^{2}=\sigma /(e-\sigma)=$ Const., i.e. if the angle of $\operatorname{grad} u$ with $V$ is constant. We can state:

Theorem 4.2. Let $f: M \rightarrow \bar{M}$ be a quasi-similarity and $V$ a submanifold of $M$. In order that the restriction of $f$ to $V$ be a quasi-similarity, it is necessary and sufficient that the field $\operatorname{grad}\left(\left|f^{\prime}\right|^{-1}\right)$ be tangent to $V$, or that it make a constant angle with $V$ and its normal component be an umbilical direction for $V$.

A special case: Hypersurfaces of an Einstein manifold. If $V$ is an hypersurface satisfying the second condition stated in Theorem 4.1, then $V$ is totally umbilical with scalar normal curvature $\lambda=(\sigma-\varrho) /|N|$. Now it is elementary to check that, in an Einstein space, the scalar normal curvature of a totally umbilical hypersurface is constant. In that case, the angular condition of Theorem 4.2 implies $|T|=$ Const. (since $(\sigma-\varrho) /|N|=$ Const.), hence $\operatorname{grad}^{2} v=2 \sigma v=$ Const., and $v=$ Const., which is realized if, and only if, $N=0$. We can state:

Theorem 4.3. Let $M$ be an Einstein space, and $u: M \rightarrow \boldsymbol{R}$ a quasi-homothetic deformation. In order that the restriction of $u$ to an hypersurface $V$ be quasi-homothetic, it is necessary and sufficient that $V$ be tangent or normal to the vector field $\operatorname{grad} u$. 
By using a previous remark, we have:

Theorem 4.4. Let $(M, g),(\bar{M}, \bar{g})$, be two Ricci-flat Riemannian manifolds of dimension $n \geqq 4, f: M \rightarrow \bar{M}$ a conformal morphism, and $V$ a flat [resp. Ricci-flat] hypersurface of $M$. In order that $f(V)$ be flat [resp. Ricci-flat] it is necessary and sufficient that the field $\operatorname{grad}\left(\left|f^{\prime}\right|^{-1}\right)$ be tangent or normal to $V$.

In particular, the only flat hypersurfaces of $\boldsymbol{R}^{n}$ whose image, under an inversion with pole 0 , is a flat hypersurface, are parts of cones with vertex 0 (this result being also true for $n=3$ ).

However these results cannot be extended to submanifolds of co-dimension $\geqq 2$, as is proved by the following counter-example which infirms an assertion of [1].

Example. Let $V$ be the sutmanifold of $\boldsymbol{R}^{2 p}$, image of the domain $\left(t_{i}>0\right)$ of $\boldsymbol{R}^{p}$ under the imbedding $f$ of components

$$
f_{k}\left(t_{1}, \ldots, t_{p}\right)=\frac{1}{\sqrt{2}} t_{k} \cos \left(\log t_{k}\right) \quad f_{k+p}=\frac{1}{\sqrt{2}} t_{k} \sin \left(\log t_{k}\right) \quad(1 \leqq k \leqq p) .
$$

We have $\sum_{i=1}^{2 p} d f_{i}^{2}=\sum_{k=1}^{p} d t_{k}^{2}$, which proves that $V$ is flat.

The image of $V$ under the inversion $j: x \mapsto|x|^{-2} x$ admits the parametrization $g=j \circ f$, which satisfies

$$
\sum_{i=1}^{2 p} d g_{i}^{2}=\sum_{i=1}^{2 p} \frac{d f_{i}^{2}}{|f|^{4}}=\frac{4}{|t|^{4}} \sum_{k=1}^{p} d t_{k}^{2} .
$$

So $j(V)$ is flat, although $V$ is neither tangent nor orthogonal to the field grad $\left|j^{\prime}\right|^{-1}$. In fact the tangential and normal components of $\operatorname{grad}\left|j^{\prime}(x)\right|^{-1}=2 x$ at the point $x=f(t)$ are given by:

$$
\begin{array}{lll}
N_{k}=f_{k}+f_{k+p}, & N_{k+p}=f_{k+p}-f_{k} & (1 \leqq k \leqq p) \\
T_{k}=f_{k}-f_{k+p}, & T_{k+p}=f_{k}+f_{k+p} & (1 \leqq k \leqq p) .
\end{array}
$$

We can check that $N$ is an umbilical direction for $V$, and that $\operatorname{grad}\left|j^{\prime}\right|^{-1}$ makes an angle of $\pi / 4$ with $V$. Thus conditions of Theorem 4.2 are exactly fulfilled.

\section{References}

[1] BlaIR, D. E.: On conformal images of flat submanifolds. - Geom. Dedicata 12, 1982, $205-208$.

[2] BRINkmanN, H. W.: Einstein spaces which are mapped conformally on each other. - Math. Ann. 94, 1925, 119-145.

[3] Eisenhart, L. P.: Riemannian geometry. - Princeton University Press, Princeton, 1949.

[4] Ferrand, J.: Les géodésiques des structures conformes. - C. R. Acad. Sci. Paris Sér. I Math. 294, 1982, 629-632. 
[5] Ferrand, J.: Sur une classe de morphismes conformes. - C. R. Acad. Sci. Paris Sér. I Math. 296, 1983, 681-684.

[6] IshiHARA, S., and Y. TASHIRo: On Riemannian manifolds admitting a concircular transformation. - Math. J. Okayama Univ. Y, 1959, 19-47.

[7] Lafontaine, J.: Sur la géométrie d'une généralisation de l'équation différentielle d'Obata. J. Math. Pures Appl. (9) 62, 1983, 63-72.

[8] Овата, M.: Certain conditions for a Riemannian manifold to be isometric with a sphere. J. Math. Soc. Japan 14, 1962, 333-340.

[9] TACHIBANA, S.: On concircular geometry and Riemannian spaces with constant scalar curvature. - Tôhoku Math. J. 3, 1951, 149-158.

[10] TASHIRo, Y.: Complete Riemannian manifolds and some vector fields. - Trans. Amer. Math. Soc. 117, 1965, 251-275.

[11] VenzI, P.: The metric $d s^{2}=F(u) d u^{2}+G(u) d \sigma^{2}$ and an application to concircular mappings. Utilitas Math. 22, 1982, 221-233.

[12] Vogel, W. O.: Kreistreue Transformationen in Riemannschen Räumen. - Arch. Math. (Basel) $21,1970,641-645$.

[13] Yano, K.: Concircular geometry I, II, III, IV, V. - Proc. Imp. Acad. Tokyo 16, 1940, 195-200, 354-360, 505-511, and ibid. 18, 1942, 446-451.

\author{
University of Paris VI \\ Department of Mathematics \\ F-75230 Paris Cedex 05 \\ France
}

Received 2 April 1984 